\author{
Advanced Studies in Theoretical Physics \\ Vol. 10, 2016, no. 5, 201 - 222 \\ HIKARI Ltd, www.m-hikari.com \\ http://dx.doi.org/10.12988/astp.2016.6312
}

\title{
The Uncertainty Principle, Spacetime Fluctuations and Measurability Notion in Quantum Theory and Gravity
}

\author{
Alexander Shalyt-Margolin \\ Institute for Nuclear Problems, Belarusian State University \\ 11 Bobruiskaya str., Minsk 220040, Belarus \\ Copyright (c) 2016 Alexander Shalyt-Margolin. This article is distributed under the \\ Creative Commons Attribution License, which permits unrestricted use, distribution, and \\ reproduction in any medium, provided the original work is properly cited.
}

\begin{abstract}
This paper is a continuation of the works earlier published by the author and deals with the theories involving a minimal length at all energy scales. The previously introduced notion of measurability is also used. As a new step, in this work at the initial stage possible contributions from the inclusion of the space-time quantum fluctuations into quantum theory and gravity are studied.
\end{abstract}

PACS: 03.65, 05.20

Keywords: minimal length, measurability, quantum theory, gravity

\section{Introduction. Main Motivation and Aim}

This paper directly continuous the earlier studies of the author [1]-[6] and in part [7]. The principal idea that has been put forward in [6] is further developed in this paper as follows: due to the Uncertainty Principle, in quantum theory there are solid grounds to consider as a background space not continuous space-time but discrete space-time involving the minimal length $l_{\text {min }}$ and the minimal time $t_{\text {min }}$. Such a space represents a lattice but very irregular lattice in a sense that all its variations are determined by the existent energies. 
At the present time all the fundamental theories at the well-known energy scales (gravity, quantum theory, statistical physics, and the like) are associated with continuous space-time and with the corresponding mathematical apparatus of infinitesimal space-time variations (increments) $d x_{\mu}, \delta x_{\mu}, d s, \delta s, \ldots$ Until very recently, this apparatus has been well applicable both in classical and quantum physics. However, in the first case the mathematical apparatus has been used without difficulties, whereas in the quantum case its use provokes various problems: (1) the problem of ultraviolet and infrared divergences; (2) the problem of the gravity non-renormalizability in a quantum approach, and (3) the problem, that is more general, of the adequate transition to the ultraviolet limit in quantum gravity.

The efforts to solve these problems in theoretical physics have generated numerous attractive and important approaches: supersymmetry, supergravity, superstrings, $M$-theories, and so on (for example, [8]).

But all the mentioned theories are actualized at high (Plancks) energies $E \approx$ $E_{P}$. At low energies $E \ll E_{P}$ they, to a high accuracy, should lead to the well-known Quantum Theory (QT) [9], [10] and General Relativity (GR) [11] defined for the continuous space-time.

In the majority of the above-mentioned approaches high (Plancks) energies $E \approx E_{P}$ are associated with the minimal length $l_{\min } \propto l_{P}$ that disappears at low energies $E \ll E_{P}$, i.e. $l_{\min } \rightarrow 0$.

But if $l_{\min }$ is really present, it must be present at all the "Energy Levels" of the theory, low energies including. Therefore, in this case the mathematical formalism of the theory should not involve any infinitesimal spatial-temporal quantities. Besides, some new parameters become involved, which are dependent on $l_{\min }[12]-[21]$. But, on the other hand, these parameters could hardly disappear totally at low energies, i.e., for QT and GR too. However, since the well-known canonical statement of QT [9], [10] and GR [11] has no such parameters, the inference is as follows: their influence at low energies is so small that it may be disregarded at the modern stage in evolution of the theory and of the experiment.

Still this does not imply that they should be ignored in future evolution of the theory, especially on going to its high-energy limit.

In this way this paper, similarly to the cited works [1]-[6], has been motivated by the need for actualization of the minimal length $l_{\text {min }}$ and the minimal time $t_{\min }$, and also of their associated parameters at all the Energy Levels of the theory, low energies $\left(E \ll E_{P}\right)$ including. It should be noted that the inclusion of $l_{\min }\left(t_{\min }\right)$ is dictated not only by selection of the corresponding model but by the application of the fundamental principles of quantum theory within the scope of quite natural assumptions ([6] and Section 2). 
It is clear that, because of the actualization of $l_{\min }\left(t_{\min }\right)$ at all the Energy Levels, the theory is specified for discrete space-time rather than for continuous space-time. By the main hypothesis set up by the author, the adequately resolved discrete theory should have the following properties:

a) at low energies, which are far from the Plank energies $E \ll E_{P}$, this theory is very close to the initial continuous theory;

b) the problems indicated in points (1)-(3) in this theory will be solved naturally (without the appearance of infinities) within the scope of the transition from low to high energies $E \approx E_{P}$ and vice versa.

The primary objective of the author is to suggest the adequate derivation of such discrete theory on the basis of his previous works [1]-[6]. As compared to [1]-[6], in this paper the author begins to analyze the inferences of the inclusion of space-time quantum fluctuations into quantum theory using the suggested formalism.

The paper is organized as follows. In Sections 2 and 3 the earlier authors results published in [1]-[6] are very briefly considered to give the reader deeper insight into the problem. The newly obtained results associated with inclusion of the space-time quantum fluctuations are presented in Section 4. Finally, in Conclusion the principal directions for further studies on the basis of the latest authors findings are indicated.

\section{Uncertainty Principle, Principle of Bounded Space-Time Variations (Increments) and Mea- surability}

The present study is based on two initial, simple and quite natural, suppositions $[1]-[6]$ :

I. Any small variation increment $\widetilde{\Delta} x_{\mu}$ of any spatial coordinate $x_{\mu}$ of the arbitrary point $x_{\mu}, \mu=1, \ldots, 3$ in some space-time system $R$ may be realized in the form of the uncertainty (standard deviation) $\Delta x_{\mu}$ when this coordinate is measured within the scope of Heisenberg's Uncertainty Principle (HUP) [22]

$$
\widetilde{\Delta} x_{\mu}=\Delta x_{\mu}, \Delta x_{\mu} \simeq \frac{\hbar}{\Delta p_{\mu}}, \mu=1,2,3
$$

for some $\Delta p_{\mu} \neq 0$.

Similarly, for $\mu=0$ for pair "time-energy" $(t, E)$, any small variation (in-

crement) in the value of time $\widetilde{\Delta} x_{0}=\widetilde{\Delta} t_{0}$ may be realized in the form of the uncertainty (standard deviation) $\Delta x_{0}=\Delta t$ and then

$$
\widetilde{\Delta} t=\Delta t, \Delta t \simeq \frac{\hbar}{\Delta E}
$$


for some $\Delta E \neq 0$. Here HUP is given for the nonrelativistic case. In the relativistic case HUP has the distinctive features [23] which, however, are of no significance for the general formulation of Any small variation (increment) $\widetilde{\Delta} x_{\mu}$ of any spatial coordinate $x_{\mu}$ of the arbitrary point $x_{\mu}, \mu=1, \ldots, 3$ in some space-time system $R$ may be realized in the form of the uncertainty (standard deviation) $\Delta x_{\mu}$ when this coordinate is measured within the scope of Heisenberg's Uncertainty Principle (HUP)

$$
\widetilde{\Delta} x_{\mu}=\Delta x_{\mu}, \Delta x_{\mu} \simeq \frac{\hbar}{\Delta p_{\mu}}, \mu=1,2,3
$$

for some $\Delta p_{\mu} \neq 0$. Similarly, for $\mu=0$ for pair "time-energy" $(t, E)$, any small variation (increment) in the value of time $\widetilde{\Delta} x_{0}=\widetilde{\Delta} t_{0}$ may be realized in the form of the uncertainty (standard deviation) $\Delta x_{0}=\Delta t$ and then

$$
\widetilde{\Delta} t=\Delta t, \Delta t \simeq \frac{\hbar}{\Delta E}
$$

for some $\Delta E \neq 0$. Here HUP is given for the nonrelativistic case. In the relativistic case HUP has the distinctive features [23] which, however, are of no significance for the general formulation of $\mathbf{I}$., being associated only with particular alterations in the right-hand side of the second relation Equation (3).

It is clear that at low energies $E \ll E_{P}$ (momentums $P \ll P_{p l}$ ) I. sets a lower bound for the variations (increments) $\widetilde{\Delta} x_{\mu}$ of any space-time coordinate $x_{\mu}$.

At high energies $E$ (momentums $P$ ) this is not the case if $E(P)$ have no upper limit. But, according to the modern knowledge, $E(P)$ are bounded by some maximal quantities $E_{\max },\left(P_{\max }\right)$

$$
E \leq E_{\max }, P \leq P_{\max },
$$

where in general $E_{\max }, P_{\max }$ may be on the order of Planck quantities $E_{\max } \propto$ $E_{P}, P_{\max } \propto P_{p l}$ and also may be the trans-Planck's quantities.

In any case the quantities $P_{\max }$ and $E_{\max }$ lead to the introduction of the minimal length $l_{\min }$ and of the minimal time $t_{\min }$.

II. There is the minimal length $l_{\min }$ as a minimal measurement unit for all quantities having the dimension of length, whereas the minimal time $t_{\min }=$ $l_{\text {min }} / c$ as a minimal measurement unit for all quantities having the dimension of time, where $c$ is the speed of light.

$l_{\min }$ and $t_{\min }$ are naturally introduced as $\Delta x_{\mu}, \mu=1,2,3$ and $\Delta t$ in Equations (3) and (4) for $\Delta p_{\mu}=P_{\max }$ and $\Delta E=E_{\max }$.

For definiteness, we consider that $E_{\max }$ and $P_{\max }$ are the quantities on the order of the Planck quantities, then $l_{\min }$ and $t_{\min }$ are also on the order of Planck quantities $l_{\min } \propto l_{P}, t_{\min } \propto t_{P}$. 
I.,II. are quite natural in the sense that there are no physical principles with which these suppositions are inconsistent.

The combination of suppositions I, II will be called the Principle of Bounded Space-Time Variations (Increments).

Then, since in fact Suppositions I.,II. introduce the minimal length $l_{\min }$, instead of HUP, we can consider its widely known high-energy generalizationthe Generalized Uncertainty Principle (GUP) that naturally leads to the minimal length $l_{\min }[24]-[35]$ :

$$
\Delta x \geq \frac{\hbar}{\Delta p}+\alpha^{\prime} l_{P}^{2} \frac{\Delta p}{\hbar} .
$$

Here $\alpha^{\prime}$ is the model-dependent dimensionless numerical factor and $l_{P}$ is the Planckian length. As Equation (6) is a quadratic inequality, then it naturally leads to the minimal length $l_{\text {min }}=\xi l_{P}=2 \sqrt{ } \alpha^{\prime} l_{P}$.

As the minimal unit of measurement $l_{\min }$ is available for all the quantities $L$ having the dimensions of length, the "Integrality Condition" (IC) is the case

$$
L=N_{L} l_{\text {min }},
$$

where $N_{L}>0$ is an integer number.

In a like manner the same "Integrality Condition" (IC) is the case for all the quantities $t$ having the dimensions of time. And similar to Equation (7), we get the for any time $t$ :

$$
t \equiv t\left(N_{t}\right)=N_{t} t_{m i n}
$$

Due to (7), we have

$$
\Delta x=N_{\Delta x} l_{\text {min }}
$$

Then the transition from high to low energies in GUP, i.e. $(G U P, \Delta p \rightarrow 0)=$ $(H U P)$, is nothing else but

$$
\left(N_{\Delta x} \approx 1\right) \rightarrow\left(N_{\Delta x} \gg 1\right) .
$$

Substituting (9) into (6) and making the necessary calculations, we can see that in the general case

$$
\Delta p \equiv \Delta p_{N_{\Delta x}}=\frac{\hbar}{\left(N_{\Delta x}-\frac{1}{4 N_{\Delta} x}\right) l_{\min }} .
$$

Whereas at low energies $E \ll E_{P}$

$$
\Delta p \equiv \Delta p_{N_{\Delta x}}=\frac{\hbar}{\left.N_{\Delta x}\right) l_{\min }} .
$$

At the same time, for the corresponding energy $E$ we get

$$
\Delta E \equiv \Delta E\left(N_{t}\right)=\frac{\hbar}{\left(N_{t}-\frac{1}{4 N_{t}}\right) t_{\text {min }}}
$$


or for low energies

$$
\Delta E \equiv \Delta E\left(N_{t}\right)=\frac{\hbar}{N t_{\min }} .
$$

In the relativistic case the formulae corresponding to $(11),(13)$ have been derived in [2],[6].

Note that the above-mentioned formulae may be conveniently rewritten in terms of $l_{\min }$ with the use of the deformation parameter $\alpha_{a}$. This parameter has been introduced earlier in the papers [36]-[43] as a deformation parameter (in terms of paper [44]) on going from the canonical quantum mechanics to the quantum mechanics at Planck's scales (early Universe) that is considered to be the quantum mechanics with the minimal length (QMML):

$$
\alpha_{a}=l_{\min }^{2} / a^{2},
$$

where $a$ is the measuring scale.

In the suggested formalism it seems expedient to introduce the following definition:

\section{Definition 1 (Measurability)}

(1) Let us define the quantity having the dimensions of length $L$ or time $t$ measurable, when it satisfies the relation Equation (7 (and respectively Equation (8)).

(2) Let us define any physical quantity measurable, when its value is consistent with points (1) of this Definition.

Thus, infinitesimal changes in length (and hence in time) are impossible and any such changes are dependent on the existing energies.

The indicated formalism leads to two lattices.

1) Lattice of the space-time variation- $L a t_{S-T}$ representing, to within the known multiplicative constants, the sets of nonzero integers $N_{w} \neq 0$ and $N_{t} \neq 0$ for each of the three space variables $w \doteq x ; y ; z$ and the time variable $t$

$$
\operatorname{Lat}_{S-T} \doteq\left(N_{w}, N_{t}\right)
$$

At the low energies $E \ll E_{\max } \propto E_{P}$ the low-energy part (sublattice) $L a t_{S-T}[L E]$ of Lat $_{S-T}$ is as follows:

$$
\operatorname{Lat}_{S-T}[L E]=\left(N_{w}, N_{t}\right) \equiv\left(\left|N_{x}\right| \gg 1,\left|N_{y}\right| \gg,\left|N_{z}\right| \gg 1,\left|N_{t}\right| \gg 1\right) .
$$

At high energies $E \rightarrow E_{\max } \propto E_{P}$ we, on the contrary, have the sublattice Lat $_{S-T}[H E]$ of Lat $_{S-T}$

$$
\operatorname{Lat}_{S-T}[H E]=\left(N_{w}, N_{t}\right) \equiv\left(\left|N_{x}\right| \approx 1,\left|N_{y}\right| \approx 1,\left|N_{z}\right| \approx 1,\left|N_{t}\right| \approx 1\right) .
$$


2) The lattice momenta-energies variation Lat $_{P-E}$, which is defined within the known multiplicative constants as [6]:

$$
\operatorname{Lat}_{P-E} \doteq\left(\frac{1}{N_{w}-\frac{1}{1 / 4 N_{w}}}, \frac{1}{N_{t}-\frac{1}{1 / 4 N_{t}}}\right)
$$

where $N_{w} \neq 0, N_{t} \neq 0$ are integer numbers from Equation (16). Similar to Equation (17), we obtain the low-energy (Low Energy) part or the sublattice $\operatorname{Lat}_{P-E}[L E]$ of $\operatorname{Lat}_{P-E}$

$$
\operatorname{Lat}_{P-E}[L E] \approx\left(\frac{1}{N_{w}}, \frac{1}{N_{t}}\right),\left|N_{w}\right| \gg 1,\left|N_{t}\right| \gg 1
$$

In accordance with Equation (18), the high-energy (High Energy) part (sublattice) $\operatorname{Lat}_{P-E}[H E]$ of Lat $_{P-E}$ takes the form

$$
\operatorname{Lat}_{P-E}[H E] \approx\left(\frac{1}{N_{w}-\frac{1}{1 / 4 N_{w}}}, \frac{1}{N_{t}-\frac{1}{1 / 4 N_{t}}}\right),\left|N_{w}\right| \rightarrow 1,\left|N_{t}\right| \rightarrow 1
$$

The general problem formulated in [1]-[6] is to resolve a quantum theory and gravity in terms of "measurable quantities" in the sense of Definition 1 (Measurability) given in this Section.

Because of this, in what follows all the considerations are given in terms of "measurable quantities" in the sense of Definition 1.

\section{Measurable Quantities in Quantum Theory. Start}

For convenience, we denote the minimal length $l_{\text {min }} \neq 0$ by $\ell$.

In [6] the author begins his study of the wave function in terms of measurable quantities.

Specifically, the scalar product $\langle\psi \mid \phi\rangle$ is considered in the momentum representation. And in this case it should be considered at the lattice $L_{a-E}$ from the previous Section.

What is implied?

The scalar product $\langle\psi \mid \phi\rangle$ from [34] is given by

$$
\langle\psi \mid \phi\rangle=\int_{-\infty}^{+\infty} \frac{d p}{1+\beta p^{2}} \psi^{*}(p) \phi(p)
$$

where $\beta=\ell^{2} / \hbar^{2}$.

In the case under study (22) at the low energies $E \ll E_{P}\left(\right.$ or $1 \ll\left|N_{p}\right| \leq \widetilde{\mathbf{N}}<$ 
$\infty)$ is replaced by the sum

$$
\begin{aligned}
& \langle\psi \mid \phi\rangle_{1 \ll\left|N_{p}\right| \leq \widetilde{\mathbf{N}}}=\sum_{1 \ll\left|N_{p}\right| \leq \widetilde{\mathbf{N}}} \frac{\Delta_{p}\left(p_{N}\right) \psi^{*}\left(p_{N}\right) \phi\left(p_{N}\right)}{\left(1+\frac{1}{\left(N_{p}-\frac{1}{4 N_{p}}\right)^{2}}\right)} \approx \\
& \approx \sum_{1 \ll\left|N_{p}\right| \leq \widetilde{\mathbf{N}}} \frac{\Delta_{p}\left(p_{N}\right) \psi^{*}\left(p_{N}\right) \phi\left(p_{N}\right)}{\left(1+\frac{1}{N_{p}^{2}}\right)} .
\end{aligned}
$$

Here

$$
\begin{array}{r}
\Delta_{p} p_{N}=p_{N}-p_{N+1} ; \Delta_{p}^{-1} \psi\left(p_{N}\right)=\frac{\psi\left(p_{N}\right)-\psi\left(p_{N+1}\right)}{p_{N}-p_{N+1}}= \\
=\frac{\psi\left(p_{N+1}+\Delta_{p} p_{N}\right)-\psi\left(p_{N+1}\right)}{\Delta_{p} p_{N}}
\end{array}
$$

and $\widetilde{\mathbf{N}}$ is derived from a minimum variation of the momentum $\Delta p_{\min }$ (formula (64) in [6])

Besides, in [6] it is shown that, since at low energies $\left|N_{p}\right| \gg 1$, in fact $p_{N}$ is varying continuously and, proceeding from the above formulae, we can assume that to a high accuracy the function $\phi\left(p_{N}\right),\left(\psi^{*}\left(p_{N}\right)\right)$ is differentiable in terms of this variable.

At high energies $E \approx E_{P}$ (or $\left.\left|N_{p}\right| \approx 1\right)$ (22) is replaced by the following sum:

$$
\langle\psi \mid \phi\rangle_{\left|N_{p}\right| \approx 1}=\sum_{\left|N_{p}\right| \approx 1} \frac{\Delta_{p}\left(p_{N}\right) \psi^{*}\left(p_{N}\right) \phi\left(p_{N}\right)}{\left(1+\frac{1}{\left(N_{p}-\frac{1}{4 N_{p}}\right)^{2}}\right)} .
$$

Then at all the energy scales $\langle\psi \mid \phi\rangle_{N_{p}}$ may be formally represented as follows:

$$
\langle\psi \mid \phi\rangle_{N_{p}}=\langle\psi \mid \phi\rangle_{1 \ll\left|N_{p}\right| \leq \widetilde{\mathbf{N}}}+\langle\psi \mid \phi\rangle_{\left|N_{p}\right| \approx 1}
$$

Just this approach was used to study $\langle\psi \mid \phi\rangle$ in [34], as is evidenced by the formula in (22).

However, with the formalism and terms proposed in this work, and also with the use of the formula (10) that in this case takes the form

$$
\left(\left|N_{p}\right| \approx 1\right) \rightarrow\left(1 \ll\left|N_{p}\right| \leq \widetilde{\mathbf{N}}\right),
$$

it seems more logical to consider the two components in Equation (26) separately, the first component originating in the process of the low-energy transition from the second component as follows:

$$
\langle\psi \mid \phi\rangle_{\left|N_{p}\right| \approx 1} \stackrel{\left|N_{p}\right| \gg 1}{\Rightarrow}\langle\psi \mid \phi\rangle_{1 \ll\left|N_{p}\right| \leq \widetilde{\mathbf{N}}} .
$$


We will return to the substantiation (28) in the next Section that is of primary importance for this work.

At the end of this Section it is appropriate to note the following [2],[6]:

3.1. The first part of formula (13) from [34] holds as well in the general case for each of the components in Equation (26)

$$
\langle(\psi \mid \mathbf{p}) \mid \phi\rangle=\langle\psi \mid(\mathbf{p} \mid \phi)\rangle
$$

The second part of formula (13) from [34]

$$
\langle(\psi \mid \mathbf{x}) \mid \phi\rangle=\langle\psi \mid(\mathbf{x} \mid \phi)\rangle
$$

takes place (to a high accuracy) only for the low-energy case $1 \ll\left|N_{p}\right| \leq \widetilde{\mathbf{N}}<$ $\infty$, i.e., for the first component in Equation (26).

3.2.In the last few years in a series of works [45]-[48] it has been demonstrated that within the scope of GUP fairly complex high-energy generalizations of the Standard Dispersion Relations [23] are possible, which are known as Modified Dispersion Relations (MDRs). MDRs can change only the high-energy part of the lattice $\operatorname{Lat}_{P-E}$, i.e. $\operatorname{Lat}_{P-E}[H E]$.

However, in [2],[6], as distinct from [45]-[48], the author uses the simplest (earlier) variant of GUP [24]-[33], involving a minimal length but not a minimal momentum.

\section{Curvature and Space-Time Quantum Fluc- tuations in Quantum Theory and Gravity}

Now we consider the components (26) separately (as this seems more correct) and try to substantiate the low-energy transition (28).

In the previous Section (similar to [34]) the background space curvature has not been treated implicitly it has been implied that quantum theory is considered in flat space-time. This assumption is sufficiently correct at low energies which are far from the Planck energies $E \ll E_{P}$. In this case the space-time curvature may be disregarded as the modern experimental cosmology demonstrates that the space-time geometry at the well-known energies $E \ll E_{P}$ is a geometry of flat space to a high accuracy [49].

However, at high energies $E \approx E_{P}$ this space is different from the flat space and there is no possibility to disregard this fact. According to the present-day knowledge, at Plancks scales the space exhibits high Space-Time Quantum Fluctuations (STQF) of the fundamental quantities: length, time, metric, and so on [50]-[72]. 
Let us briefly revert to STQF. The definition (STQF) is closely associated with the notion of space-time foam. The notion space-time foam, introduced by J. A. Wheeler about 60 years ago for the description and investigation of physics at Plancks scales (Early Universe) [50],[51], is fairly settled. Despite the fact that in the last decade numerous works have been devoted to physics at Plancks scales within the scope of this notion, for example [52]-[71], by this time still their no clear understanding of the space-time foam as it is. In accordance with the modern concepts, the space-time foam [51] notion forms the basis for space-time at Plancks scales (Big Bang). This object is associated with the quantum fluctuations generated by uncertainties in measurements of the fundamental quantities, inducing uncertainties in any distance measurement. A precise description of the space-time foam is still lacking along with an adequate quantum gravity theory. But for the description of quantum fluctuations we have a number of interesting methods (for example, [72],[61]-[71]). In what follows, we use the terms and symbols from [63]. Then for the fluctuations $\widetilde{\delta} l$ of the distance $l$ we have the following estimate:

$$
(\widetilde{\delta} l)_{\gamma} \gtrsim l_{P}^{\gamma} l^{1-\gamma}=l_{P}\left(\frac{l}{l_{P}}\right)^{1-\gamma}=l\left(\frac{l_{P}}{l}\right)^{\gamma}=l \lambda_{l}^{\gamma},
$$

or that same

$$
\left|(\widetilde{\delta} l)_{\gamma}\right|_{\text {min }}=\beta l_{P}^{\gamma} l^{1-\gamma}=\beta l_{P}\left(\frac{l}{l_{P}}\right)^{1-\gamma}=\beta l \lambda_{l}^{\gamma},
$$

where $0<\gamma \leq 1$, coefficient $\beta$ is of order 1 and $\lambda_{l} \equiv l_{P} / l$.

From (31),(32), we can derive the quantum fluctuations for all the primary characteristics, specifically for the time $(\tilde{\delta} t)_{\gamma}$, energy $(\widetilde{\delta} E)_{\gamma}$, and the metrics $\left(\widetilde{\delta} g_{\mu \nu}\right)_{\gamma}$. In particular, for $\left(\widetilde{\delta} g_{\mu \nu}\right)_{\gamma}$ we can use formula (10) in [63]

$$
\left(\widetilde{\delta} g_{\mu \nu}\right)_{\gamma} \gtrsim \lambda^{\gamma} .
$$

But due to GUP (6), in the case under consideration the theory involves a minimal length on the order of the Planck length

$$
\ell \propto l_{P}
$$

or that is the same

$$
\ell=\xi l_{P},
$$

where the coefficient $\xi$ is on the order of unity too.

Evidently,that in this case replacement of Plancks length by the minimal length in all the above formulae is absolutely correct and is used without detriment to the generality $[7],[1]$

$$
l_{P} \rightarrow \ell
$$


Thus, $\lambda_{l} \equiv l_{\text {min }} / l$ and then (31)- (33) upon the replacement (35) are read unchanged.

So, (32) may be written as

$$
\left|(\tilde{\delta} l)_{\gamma}\right|_{\min }=\beta l \lambda_{l}^{\gamma}=\beta N_{l}\left(N_{l}^{-\gamma}\right) \ell=\beta N_{l}^{1-\gamma} \ell .
$$

Here one should take into account the following consideration: due to the (Integrality Condition) (7) in the right-hand side of (36) for the factor $\beta N_{l}^{1-\gamma}$ before $\ell$ its integer part is always meant

$$
\beta N_{l}^{1-\gamma} \mapsto\left[\beta N_{l}^{1-\gamma}\right]
$$

and this goes without special mentioning for the whole text.

As noted in the overview [63], the value $\gamma=2 / 3$ derived in [72] is totally consistent with the Holographic Principle [73]-[76].

The following points of importance should be noted [7],[1]:

4.1)It is clear that at Plancks scales, i.e. at the minimal length scales

$$
l \rightarrow \ell
$$

models for different values of the parameter $\gamma$ are coincident.

4.2) In fact, the parameter $\lambda_{l}$ is nothing else but

$$
\lambda_{l}=\sqrt{\alpha_{l}},
$$

where $\alpha_{l}$ is defined in formula (15) for $a=l$.

It is important that the parameter $\alpha_{l}$ initially introduced in [36]-[43] is not given at the limiting point $l=l_{\text {min }}$ due to the appearance of singularity [37] and hence we have

$$
0<\alpha_{l} \leq 1 / 4
$$

It is obvious that nothing precludes $\lambda_{l}$ to be variable over the interval

$$
0<\lambda_{l} \leq 1
$$

At the same time, for complete conformity to the domain of definition (41) and to the formula of (39), at the limiting point $l=\ell$ the parameter $\alpha_{l}$ may be redefined (regularized).

It should be noted that the parameter $\alpha_{l}$ has the following clear physical meaning:

$$
\alpha_{l}^{-1} \sim S^{B H},
$$

where

$$
S^{B H}=\frac{A}{4 l_{p}^{2}}
$$


is the well-known Bekenstein-Hawking formula for the black hole entropy in the semiclassical approximation [77],[78] for the black-hole event horizon surface $A$, with the characteristics linear dimension (radius) $R=l$. This is especially obvious in the spherically-symmetric case.

Reverting to the beginning of this Section, we can state the following: as background spaces of the first and of the second components in (26) have absolutely different curvatures (the space is nearly flat for the first component and has a higher curvature for the second component), it is better to consider these components separately, the transition (28) being absolutely natural.

Considering this, the transition (28) from high to low energies may be given differently as a transition from the high-curvature background space $K \gg 0$ to the asymptotically flat space [11]

$$
\langle\psi \mid \phi\rangle_{K \gg 0} \stackrel{K_{\rightrightarrows}}{\Rightarrow}\langle\psi \mid \phi\rangle_{K \approx 0} .
$$

As this takes place, the curvature $K$ in (44) is understood as the Gaussian curvature $K(l)[79]$ corresponding to the scale $l$ :

$$
K \equiv K(l)=\frac{1}{l^{2}}=\frac{1}{N_{l}^{2} \ell^{2}}=\alpha_{l} \ell^{-2} .
$$

Because of this, the transition (44) is in complete conformity with the formula (27) from the previous Section.

The problem is, which models for space-time foam at the Planck scale adequately agree with the transition (44).

It is clear that this feature is attributed to the models considered by Fabio Scardigli in [56]-[58] and based on micro-black holes with the radius $r$ that equals several Plancks lengths $l_{P}$ or, in much the same way, several minimal lengths $\ell$ (within the scope of this paper) $r=N_{r} \ell$, where $N_{r}$-integer number on the order of 1.

Then, due to (45), in fact the transition (44) for $l=r$ takes the form

$$
\langle\psi \mid \phi\rangle_{\frac{1}{N_{r}^{2}} \gg 0} \stackrel{N_{r} \rightarrow \infty}{\Rightarrow}\langle\psi \mid \phi\rangle_{\frac{1}{N_{r}^{2}} \approx 0} .
$$

Is it possible to correct the results of the previous Section due to STQF?

4.3) At high (Plancks) energies, according to 6.1) and the formula of (38), all fluctuations of the length $l$ have the characteristic dimension $\approx \ell$. Because of this, we should take into consideration all components of the sum

$$
\langle\psi \mid \phi\rangle_{\left|N_{p}\right| \approx 1}=\sum_{\left|N_{p}\right| \approx 1} \frac{\Delta_{p}\left(p_{N}\right) \psi^{*}\left(p_{N}\right) \phi\left(p_{N}\right)}{\left(1+\frac{1}{\left(N_{p}-\frac{1}{4 N_{p}}\right)^{2}}\right)} .
$$


from the right-hand side of (25).

4.4)The situation is cardinally different at low energies $E \ll E_{P}$. According to formulae (32),(36) and considering (37), in the sum from the right-hand side of the formula (23)

$$
\begin{aligned}
\langle\psi \mid \phi\rangle_{1 \ll\left|N_{p}\right| \leq \widetilde{\mathbf{N}}} & \sum_{1 \ll\left|N_{p}\right| \leq \widetilde{\mathbf{N}}} \frac{\Delta_{p}\left(p_{N}\right) \psi^{*}\left(p_{N}\right) \phi\left(p_{N}\right)}{\left(1+\frac{1}{\left(N_{p}-\frac{1}{4 N_{p}}\right)^{2}}\right)} \approx \\
& \approx \sum_{1 \ll\left|N_{p}\right| \leq \widetilde{\mathbf{N}}} \frac{\Delta_{p}\left(p_{N}\right) \psi^{*}\left(p_{N}\right) \phi\left(p_{N}\right)}{\left(1+\frac{1}{N_{p}^{2}}\right)} .
\end{aligned}
$$

we select not all the components but only those corresponding to the points lattice "sites" Lat $_{P-E}[L E]$ from Section 3 defined by (36), (37) In this case the lattice spacing in the sum from the right-hand side of (48) is not single and equal to $\ell$, being variable, determined by the scale $l$, and dependent on the energies present

$$
\widetilde{N}_{l} \ell=\left[\beta N_{l}^{1-\gamma}\right] \ell
$$

where $\beta, \gamma$ is taken from formulae (32),(36),(37).

The situation is quite natural. Indeed, the transition (48) from the point with the number $N$ to the point with the number $N+1$ means that the length $l=\ell$ corresponding to the "difference" of these points is a measurable quantity. By Definition 1, the minimal length $\ell$ is actually a measurable quantity but only for the energies $E \approx E_{P}$, and in this point we consider the case $E \ll E_{P}$.

It should be noted that STQF must be taken into account in gravity too.

Let us consider an example of the static spherically-symmetric horizon space that has been treated in [80] and subsequently studied by the author in [81], and also in [1].

The metric on the horizon of this space is of the form [80]

$$
d s^{2}=-f(r) c^{2} d t^{2}+f^{-1}(r) d r^{2}+r^{2} d \Omega^{2} .
$$

The horizon location will be given by a simple zero of the function $f(r)$, at the radius $r=a$.

In [1] it has been demonstrated that the Einstein equation on horizon in this case may be written in terms of the Gaussian curvature(see the above-given formula (45)) as

$$
\frac{c^{4}}{G}\left(-f^{\prime}\left(K_{a}\right) K_{a}^{2}-\frac{1}{2} K_{a}\right)=4 \pi P\left(K_{a}\right)
$$

where $K_{a}$ is the Gaussian curvature corresponding to the horizon radius $r=a$; $P\left(K_{a}\right)=T_{a}^{a}$ is the trace of the momentum-energy tensor and radial pressure. 
Then, in analogy with 4.3) and 4.4), we obtain

4.5) At high energies $E \approx E_{P}$ in the case under study the radius of this space is close to the minimal length $\ell, a \approx \ell$ in analogy with [56]-[58] micro-black holes having the radius $r=a$ [81], [1]. All STQF of $r=a$ will be $\approx \ell$, as in 4.3.The Gaussian curvature $K_{a}(45)$ ) becomes enormous, drastically varying for any change of the space radius (formulae (46),(47) in [1]).

4.6) At low energies $E \ll E_{P}$, and vice versa $r=a \gg \ell$, the Gaussian curvature $K_{a}=1 / a^{2} \approx 0$ takes a (nonuniform) discrete series of values close to zero. $K_{a}$, as distinct from (4.5), is varying smoothly. Besides, STQF of the radius $r=a$ will be $\gg \ell$. They are given by the formula of (49) for $l=a$, as noted in [1] (formula (35)).

Of course, Section 4 of this paper and the results of the previous works [1]-[6] are only the first steps to resolve the Quantum Theory and Gravity in terms of measurable quantities using Definition 1. It is necessary to study thoroughly the low-energy case $E \ll E_{P}$ and the correct transition to high energies $E \propto E_{P}$ taking into account STQF. The author is planning to treat these problems in his further works.

\section{Conclusion}

In several works $[2,6]$ the main points have been formulated and the problems associated with the suggested approach have been indicated. They may be concluded as follows.

5.1 When in the theory the minimal length $l_{\min } \neq 0$ is actualized (involved) at all the energy scales, a mathematical apparatus of this theory must be changed considerably: no infinitesimal space-time variations (increments) must be involved, the key role being played by the definition of measurability (Definition 1 from Section 2).

5.2 As this takes place, the theory becomes discrete at all the energy scales but at low energies (far from the Planck energies) the sought for theory must be very close in its results to the starting continuous theory (with $l_{\min }=0$ ). In the process a real discreteness is exhibited only at high energies which are close to the Planck energies.

5.3 By this approach the theory at low and high energies is associated with a common single set of the parameters $\left(N_{L}\right.$ from Formula $\left.(7)\right)$ or with the dimensionless small parameters $\left(1 / N_{L}=\sqrt{ } \alpha_{L}\right)$ which are lacking if at low energies the theory is continuous, i.e., when $l_{\min }=0$. 
The principal objective of my further studies is to develop for quantum theory and gravity, within the scope of the considerations given in points $\mathbf{5 . 1}-\mathbf{5 . 3}$, the corresponding discrete models (with $l_{\text {min }} \neq 0$ ) for all the energy scales and to meet the following requirements:

5.4 At low energies the models must, to a high accuracy, represent the results of the corresponding continuous theories.

5.5 The models should not have the problems of transition from low to high energies and, specifically, the ultraviolet divergences problem. By author's opinion, the problem associated with points $\mathbf{5 . 4}$ and $\mathbf{5 . 5}$ is as follows.

5.6 It is interesting to know why, with the existing $l_{\text {min }} \neq 0, t_{\text {min }} \neq 0$ and discreteness of nature, at low energies $E \ll E_{\max } \propto E_{P}$ the apparatus of mathematical analysis based on the use of infinitesimal space-time quantities

$\left(d x_{\mu}, \frac{\partial \varphi}{\partial x_{\mu}}\right.$, and so on) is very efficient giving excellent results. The answer is simple: in this case $l_{\min }$ and $t_{\min }$ are very far from the available scale of $L$ and $t$, the corresponding $N_{L} \gg 1, N_{t} \gg 1$ being in general true but insufficient. There is a need for rigorous calculations.

Based on Section 4 of the present paper, the points $\mathbf{5 . 1 - 5 . 6}$ should be supplemented with points $\mathbf{5 . 7}-\mathbf{5 . 9}$.

5.7 Is the exponent of $\gamma$ in formulae (31),(32), and so on constant $\gamma \equiv$ const. or is it dependent on the existent energies $\gamma=\gamma(E)$ ?

5.8 The Gaussian curvature $K(r)$ considered in Section 4 leads to the simplest geometry that, to a high accuracy, is flat at low energies. Besides, it is readily expressed in terms of measurable quantities.

The problem is to suggest a constructive description for a maximally wide class of the geometries which are asymptotically flat in the limit of low energies $E \ll E_{P}$. In this context the word constructive is used in terms of measurable quantities. Solving of this problem is directly associated with the constructive description of space-time at Planck's scale or of space-time foam.

5.9 And, finally, is there a direct relation between the preceding point and the expression of the main gravity components $R_{\mu}^{\nu}\left(\alpha_{l}\right), R\left(\alpha_{l}\right), T_{\mu}^{\nu}\left(\alpha_{l}\right), \Lambda\left(\alpha_{l}\right)$ from Section 4 in terms of measurable quantities?

\section{Conflict of Interests}

The author declares that there is no conflict of interests regarding the publication of this article. 


\section{References}

[1] A.E. Shalyt-Margolin, Minimal Length and the Existence of Some Infinitesimal Quantities in Quantum Theory and Gravity, Adv. High Energy Phys., 2014 (2014), 1-8. http://dx.doi.org/10.1155/2014/195157

[2] A.E. Shalyt-Margolin, Holographic Principle, Minimal Length and Measurability, J. Adv. Phys., 5 (2016), no. 3, 263-275.

http://dx.doi.org/10.1166/jap.2016.1274

[3] Alexander Shalyt-Margolin, Minimal Length, Measurability, Continuous and Discrete Theories, Chapter 7 in Horizons in World Physics, Volume 284, A. Reimer, Ed., Nova Science, Hauppauge, New York, USA, 2015, 213-229.

[4] Alexander Shalyt-Margolin, Chapter 5 in Advances in Dark Energy Research, Miranda L. Ortiz, Ed., Nova Science, Hauppauge, New York, USA, 2015, 103-124.

[5] Alexander Shalyt-Margolin, Minimal Length at All Energy Scales and Measurability, Nonlinear Phenomena in Complex Systems, 19 (2016), no. 1 , (in press).

[6] Alexander Shalyt-Margolin, Minimal Length, Measurability and Gravity, Entropy, 18 (2016), no. 3, 80. http://dx.doi.org/10.3390/e18030080

[7] A.E. Shalyt-Margolin, Space-Time Fluctuations, Quantum Field Theory with UV-cutoff and Einstein Equations, Nonlinear Phenomena in Complex Systems, 17 (2014), no. 2, 138-146.

[8] Joseph Polchinski, String Theory, Vol. I, II, Cambridge University Press, 1998. http://dx.doi.org/10.1017/cbo9780511816079 http://dx.doi.org/10.1017/cbo9780511618123

[9] A. Messiah, Quantum Mechanics, Volumes 1, 2, North Holland Publishing Company, Amsterdam, The Netherlands, 1967.

[10] M.E. Peskin, D.V. Schroeder, An Introduction to Quantum Field Theory, Addison-Wesley Publishing Company, 1995.

[11] R.M. Wald, General Relativity, University of Chicago Press, Chicago, USA, 1984. http://dx.doi.org/10.7208/chicago/9780226870373.001.0001

[12] G. Amelino-Camelia, Quantum Spacetime Phenomenology, Living Rev. Relativ., 16 (2013), no. 2, 5-129. http://dx.doi.org/10.12942/lrr-2013-5 
[13] R. Penrose, Quantum Theory and Space-Time, Chapter in The Nature of Space and Time, Prinseton University Press, Princeton, New Jersey, USA, 1996.

[14] L. Garay, Quantum gravity and minimum length, Int. J. Mod. Phys. A, 10 (1995), 145-165. http://dx.doi.org/10.1142/s0217751x95000085

[15] G. Amelino-Camelia, L. Smolin, Prospects for constraining quantum gravity dispersion with near term observations, Phys. Rev. D, 80 (2009). http://dx.doi.org/10.1103/physrevd.80.084017

[16] G. Gubitosi et al., A constraint on planck-scale modifications to electrodynamics with CMB polarization data, J. Cosmol. Astropart. Phys., 2009 (2009), 21-34. http://dx.doi.org/10.1088/1475-7516/2009/08/021

[17] G. Amelino-Camelia, Building a case for a planck-scale-deformed boost action: The planck-scale particle-localization limit, Int. J. Mod. Phys. D, 14 (2005), 2167-2180. http://dx.doi.org/10.1142/s0218271805007978

[18] S. Hossenfelder et al., Signatures in the Planck Regime, Phys. Lett. B, 575 (2003), 85-99. http://dx.doi.org/10.1016/j.physletb.2003.09.040

[19] S. Hossenfelder, Running Coupling with Minimal Length, Phys. Rev. D, 70 (2004). http://dx.doi.org/10.1103/physrevd.70.105003

[20] S. Hossenfelder, A note on theories with a minimal length, Class. Quantum Gravity, 23 (2006), 1815-1821. http://dx.doi.org/10.1088/0264-9381/23/5/n01

[21] S. Hossenfelder, Minimal Length Scale Scenarios for Quantum Gravity, Living Rev. Relativ., 16 (2013). http://dx.doi.org/10.12942/lrr-2013-2

[22] W. Heisenberg, Uber den anschaulichen Inhalt der quantentheoretischen Kinematik und Mechanik, Z. Phys., 43 (1927), 172-198, (In German). http://dx.doi.org/10.1007/bf01397280

[23] V.B. Berestetskii, E.M. Lifshitz, L.P. Pitaevskii, Relativistic Quantum Theory, Pergamon, Oxford, UK, 1971.

[24] G. A. Veneziano, A stringy nature needs just two constants, Europhys. Lett., 2 (1986), 199-204. http://dx.doi.org/10.1209/0295-5075/2/3/006

[25] D. Amati, M. Ciafaloni and G. A. Veneziano, Can spacetime be probed below the string size?, Phys. Lett. B, 216 (1989), 41-47. http://dx.doi.org/10.1016/0370-2693(89)91366-x 
[26] E. Witten, Reflections on the fate of spacetime, Phys. Today, 49 (1996), 24-28. http://dx.doi.org/10.1063/1.881493

[27] R. J. Adler and D. I. Santiago, On gravity and the uncertainty principle, Mod. Phys. Lett. A, 14 (1999), 1371-1381.

http://dx.doi.org/10.1142/s0217732399001462

[28] D.V. Ahluwalia, Wave-particle duality at the Planck scale: freezing of neutrino oscillations, Phys. Lett. A, 275 (2000), 31-35.

http://dx.doi.org/10.1016/s0375-9601(00)00578-8

[29] D.V. Ahluwalia, Interface of gravitational and quantum realms, Mod. Phys. Lett. A, 17 (2002), 1135-1145.

http://dx.doi.org/10.1142/s021773230200765x

[30] M. Maggiore, The algebraic structure of the generalized uncertainty principle, Phys. Lett. B, 319 (1993), 83-86.

http://dx.doi.org/10.1016/0370-2693(93)90785-g

[31] M. Maggiore, Black Hole Complementarity and the Physical Origin of the Stretched Horizon, Phys. Rev. D, 49 (1994), 2918-2921.

http://dx.doi.org/10.1103/physrevd.49.2918

[32] M. Maggiore, A Generalized Uncertainty Principle in Quantum Gravity, Physics Letters B, 304 (1993), 65-69.

http://dx.doi.org/10.1016/0370-2693(93)91401-8

[33] S. Capozziello, G. Lambiase and G. Scarpetta, Generalized Uncertainty Principle from Quantum Geometry, Int. J. Theor. Phys., 39 (2000), 1522. http://dx.doi.org/10.1023/a:1003634814685

[34] A. Kempf, G. Mangano and R.B. Mann, Hilbert space representation of the minimal length uncertainty relation, Phys. Rev. D, 52 (1995), 11081118. http://dx.doi.org/10.1103/physrevd.52.1108

[35] K. Nozari, A. Etemadi, Minimal length, maximal momentum, and Hilbert space representation of quantum mechanics, Phys. Rev. D, 85 (2012), 104029. http://dx.doi.org/10.1103/physrevd.85.104029

[36] A.E. Shalyt-Margolin, J.G. Suarez, Quantum Mechanics of the Early Universe and Its Limiting Transition, Available online: http://arxiv.org/abs/gr-qc/0302119

[37] A.E. Shalyt-Margolin, J.G. Suarez, Quantum mechanics at Planck's scale and density matrix, Int. J. Mod. Phys. D, 12 (2003), 1265-1278. http://dx.doi.org/10.1142/s0218271803003700 
[38] A.E. Shalyt-Margolin and A. Ya. Tregubovich, Deformed density matrix and generalized uncertainty relation in thermodynamics, Mod. Phys. Lett. A, 19 (2004), 71-81. http://dx.doi.org/10.1142/s0217732304012812

[39] A.E. Shalyt-Margolin, Non-unitary and unitary transitions in generalized quantum mechanics, new small parameter and information problem solving, Mod. Phys. Lett. A, 19 (2004), 391-403.

http://dx.doi.org/10.1142/s0217732304013155

[40] A.E. Shalyt-Margolin, Pure states, mixed states and Hawking problem in generalized quantum mechanics, Mod. Phys. Lett. A, 19 (2004), 20372045. http://dx.doi.org/10.1142/s0217732304015312

[41] A.E. Shalyt-Margolin, The universe as a nonuniform lattice in finitevolume hypercube I: Fundamental definitions and particular features, Int. J. Mod. Phys. D, 13 (2004), 853-863.

http://dx.doi.org/10.1142/s0218271804004918

[42] A.E. Shalyt-Margolin, The Universe as a nonuniformlattice in the finitedimensional hypercube II: Simple cases of symmetry breakdown and restoration, Int. J. Mod. Phys. A, 20 (2005), 4951-4964.

http://dx.doi.org/10.1142/s0217751x05022895

[43] A.E. Shalyt-Margolin, The density matrix deformation in physics of the early universe and some of its implications, Chapter in Quantum Cosmology Research Trends, A. Reimer, Ed., Nova Science, Hauppauge, New York, USA, 2005, 49-92.

[44] L. Faddeev, Mathematical view of the evolution of physics, Priroda, 5 (1989), 11-16.

[45] A.N. Tawfik, A. Diab, Generalized Uncertainty Principle: Approaches and Applications, Int. J. Mod. Phys. D, 23 (2014), 1430025.

http://dx.doi.org/10.1142/s0218271814300250

[46] E.C. Vagenas, B.R. Majhi, Modified Dispersion Relation, Photon's Velocity, and Unruh Effect, Phys. Lett. B., 725 (2013), 477-480.

http://dx.doi.org/10.1016/j.physletb.2013.07.039

[47] K. Nozari, A.S. Sefidgar, Comparison of Approaches to Quantum Correction of Black Hole Thermodynamics, Phys. Lett. B, 635 (2006), 156-160. http://dx.doi.org/10.1016/j.physletb.2006.02.043

[48] K. Nozari, B. Fazlpour, Generalized Uncertainty Principle, Modified Dispersion Relations and the Early Universe Thermodynamics, Gen. Relativ. 
Gravit., 38 (2006), 1661-1679.

http://dx.doi.org/10.1007/s10714-006-0331-0

[49] Steven Weinberg, Cosmology, Oxford University Press, 2008.

[50] J.A. Wheeler, Geometrodynamics, Academic Press, New York and London, 1962.

[51] C.W. Misner, K.S. Thorne and J.A. Wheeler, Gravitation, Freeman, San Francisco, 1973.

[52] Remo Garattini, A spacetime foam approach to the cosmological constant and entropy, Int. J. Mod. Phys. D, 11 (2002), 635-651.

http://dx.doi.org/10.1142/s0218271802001445

[53] Remo Garattini, Spacetime Foam Approach to the Schwarzschild-de Sitter Entropy, Entropy, 2 (2000), 26-38.

http://dx.doi.org/10.3390/e2010026

[54] Remo Garattini, Entropy and the cosmological constant: a spacetimefoam approach, Nucl. Phys. B - Proc. Suppl., 88 (2000), 297-300.

http://dx.doi.org/10.1016/s0920-5632(00)00789-1

[55] Remo Garattini, Entropy from the foam, Phys. Lett. B, 459 (1999), 461-467. http://dx.doi.org/10.1016/s0370-2693(99)00700-5

[56] Fabio Scardigli, Black Hole Entropy: a spacetime foam approach, Class. Quant. Grav., 14 (1997), 1781-1793.

http://dx.doi.org/10.1088/0264-9381/14/7/014

[57] Fabio Scardigli, Generalized Uncertainty Principle in Quantum Gravity from Micro-Black Hole Gedanken Experiment, Phys. Lett. B, 452 (1999), 39-44. http://dx.doi.org/10.1016/s0370-2693(99)00167-7

[58] Fabio Scardigli, Gravity coupling from micro-black holes, Nucl. Phys. B-Proc. Suppl., 88 (2000), 291-294.

http://dx.doi.org/10.1016/s0920-5632(00)00788-x

[59] Luis J. Garay, Thermal properties of spacetime foam, Phys. Rev. D, 58 (1998), 124015. http://dx.doi.org/10.1103/physrevd.58.124015

[60] Luis J. Garay, Spacetime foam as a quantum thermal bath, Phys. Rev. Lett., 80 (1998), 2508-2511.

http://dx.doi.org/10.1103/physrevlett.80.2508 
The uncertainty principle, spacetime fluctuations and ...

[61] Y.J. Ng and H. van Dam, Measuring the foaminess of space-time with gravity-wave interferometers, Found. Phys., 30 (2000), 795-805. http://dx.doi.org/10.1023/a:1003745212871

[62] Y.J. Ng, Spacetime foam, Int. J. Mod. Phys. D, 11 (2002), 1585-1590. http://dx.doi.org/10.1142/s0218271802002931

[63] Y.J. Ng, Selected topics in Planck-scale physics, Mod. Phys. Lett. A, 18 (2003), 1073-1097. http://dx.doi.org/10.1142/s0217732303010934

[64] Y.J. Ng, Quantum Foam, arxiv.org/abs/gr-qc/0401015.

[65] Y.J. Ng, H. van Dam, Spacetime Foam, Holographic Principle, and Black Hole Quantum Computers, Int. J. Mod. Phys. A, 20 (2005), 1328-1335. http://dx.doi.org/10.1142/s0217751x05024237

[66] W.A. Christiansen, Y. Jack Ng, H. van Dam, Probing spacetime foam with extragalactic sources, Phys. Rev. Lett., 96 (2006), 051301. http://dx.doi.org/10.1103/physrevlett.96.051301

[67] Y.J. Ng, Holographic Foam, Dark Energy and Infinite Statistics, Phys. Lett. B, 657 (2007), 10-14.

http://dx.doi.org/10.1016/j.physletb.2007.09.052

[68] Y.J. Ng, Spacetime Foam and Dark Energy, AIP Conf. Proc., 1115 (2009), 74-79. http://dx.doi.org/10.1063/1.3131531

[69] Wayne A. Christiansen, David J.E. Floyd, Y. Jack Ng, Eric S. Perlman, Limits on Spacetime Foam, Phys. Rev. D, 83 (2011), 084003. http://dx.doi.org/10.1103/physrevd.83.084003

[70] G. Amelino-Camelia, An interferometric gravitational wave detector as a quantum-gravity apparatus, Nature, 398 (1999), 216-218.

[71] L. Diosi and B. Lukacs, On the minimum uncertainties of space-time geodesics, Phys. Lett. A, 142 (1989), 331-334.

http://dx.doi.org/10.1016/0375-9601(89)90375-7

[72] E.P. Wigner, Relativistic Invariance and Quantum Phenomena, Rev. Mod. Phys., 29 (1957), 255-268.

http://dx.doi.org/10.1103/revmodphys.29.255

[73] Gerard 't Hooft, Dimensional reduction in quantum gravity, (1993), arXiv:gr-qc/9310026. 
[74] Gerard 't Hooft, The Holographic Principle, Basics and Highlights in Fundamental Physics, (2000), 72-100. http://dx.doi.org/10.1142/9789812811585_0005

[75] L. Susskind, The world as a hologram, J. Math. Physics, 36 (1995), 6377-6396. http://dx.doi.org/10.1063/1.531249

[76] R. Bousso, The Holographic principle, Rev. Mod. Phys, 74 (2002), 825874. http://dx.doi.org/10.1103/revmodphys.74.825

[77] J.D. Bekenstein, Black holes and entropy, Physical Review D, 7 (1973), 2333-2346. http://dx.doi.org/10.1103/physrevd.7.2333

[78] S.W. Hawking, Black holes and thermodynamics, Physical Review D, 13 (1976), 191-197. http://dx.doi.org/10.1103/physrevd.13.191

[79] S. Kobayashi, K. Nomozu, Foundations of Differential Geometry, Vol. II, Interscience Publishers, New York-London-Sydney, 1969.

[80] T. Padmanabhan, Thermodynamical aspects of gravity: new insights, Reports on Progress in Physics, 73 (2010), Article ID 046901. http://dx.doi.org/10.1088/0034-4885/73/4/046901

[81] A.E. Shalyt-Margolin, Quantum theory at planck scale, limiting values, deformed gravity and dark energy problem, Int. J. Mod. Phys. D, 21 (2012), 1250013. http://dx.doi.org/10.1142/s0218271812500137

Received: March 30, 2016; Published: May 15, 2016 